\title{
Review of: "Evolution of cooperation and consistent personalities in public goods games"
}

\author{
Juan $\mathrm{Li}^{1}$ \\ 1 Dalian University of Technology
}

Potential competing interests: The author(s) declared that no potential competing interests exist.

As we all know, in the repeated public goods game, the synergy factor $r(1<r<n, n$ represents the group size) supports the evolution of the cooperators, that is, the higher the $r$, the more evolutionary advantage the cooperative strategy has. In this paper, when presenting the results of the PGG with a reward dilemma, the authors should inform the results of the repeated game with only one round. Taking this as a baseline, the promotion effect of the two-stage game can be explained more clearly. In addition, in the second scenario, the assortative game, where the cooperators and defectors of the first round are in separate groups in the second round, the authors conclude that the classification game explains the evolution of consistent personality strategies. This result may be more convincing if a reference is set, that the cooperators and defectors in the first round are still in the same group in the second round of the game (similar to a stochastic game). The results of this paper will be clearer if the author can briefly explain the results under the above two baseline cases. 